\title{
A Life Threatening Complication of Anticoagulation Prophylaxis- Bilateral Adrenal Hemorrhage
}

Anitha Rajamanickam, MD Preethi Patel, MD

Prabhakaran Anbazhagan, MD

Brian Harte, mD

\begin{abstract}
Department of Hospital Medicine, Cleveland Clinic Foundation, Cleveland, Ohio.
\end{abstract}
Disclosure: Nothing to report.

Journal of Hospital Medicine 2009;4:E25-E27. @ 2009 Society of Hospital Medicine.

KEYWORDS: adrenal hemorrhage, DVT prophylaxis, low molecular weight heparin.

A 52-year-old man presented to the emergency department (ED) from a skilled nursing facility with a complaint of bilateral upper-quadrant abdominal pain of 48 hours' duration. The pain was sharp, nonradiating, constant, and was associated with nausea, vomiting, and constipation. The patient denied any fever, back pain, dysuria, melena, or hematochezia. In the rehabilitation facility the patient had been initially evaluated for this pain. He was given laxatives and stool softeners for presumed constipation but these measures had not been effective. A computed tomography (CT) scan of the abdomen had only showed stool in the colon and he was sent to the ED for further evaluation.

Apart from severe degenerative joint disease in both his knees he was in good health. He was in the skilled nursing facility (SNF) for rehabilitation for bilateral knee replacement surgery done 9 days prior to this presentation. His postoperative course was unremarkable. He had been maintained on prophylaxis for venous thromboembolism with enoxaparin since postoperative day 1 at a daily dose of $40 \mathrm{mg}$ subcutaneously, and was transferred to the SNF on postoperative day 6 on the same dose. His was receiving oxycodone and Tylenol for pain. He was on no other medications.

Vital signs on presentation revealed a temperature of $97.5^{\circ} \mathrm{F}$, a heart rate of 100 beats per minute, a respiratory rate of 16 breaths per minute, and a blood pressure of 136/ $69 \mathrm{mmHg}$. He was alert and oriented and in mild distress from the abdominal pain. Examination was normal except for tenderness in the upper quadrants of the abdomen though no rigidity or rebound tenderness were noted. Routine chemistries were normal except for sodium of $134 \mathrm{mg} /$ dL. His white count, hemoglobin, hematocrit, and platelet levels were noted to be at $17.5 \mathrm{~K} / \mu \mathrm{L}, 10 \mathrm{~g} / \mathrm{dL}, 30 \%$, and $345 \mathrm{~K} / \mu \mathrm{L}$, respectively, and were stable with regard to his discharge laboratory values. His serum eosinophil level was normal. A complete workup for hypercoagulable state and bleeding disorders including assays for antibodies associated with heparin-induced thrombocytopenia were negative. $\mathrm{He}$ was admitted for further evaluation and treatment.

The patient had another CT scan of the abdomen (Figure 1), which when compared to the one done at the SNF 2 days prior showed "markedly enlarged" bilateral

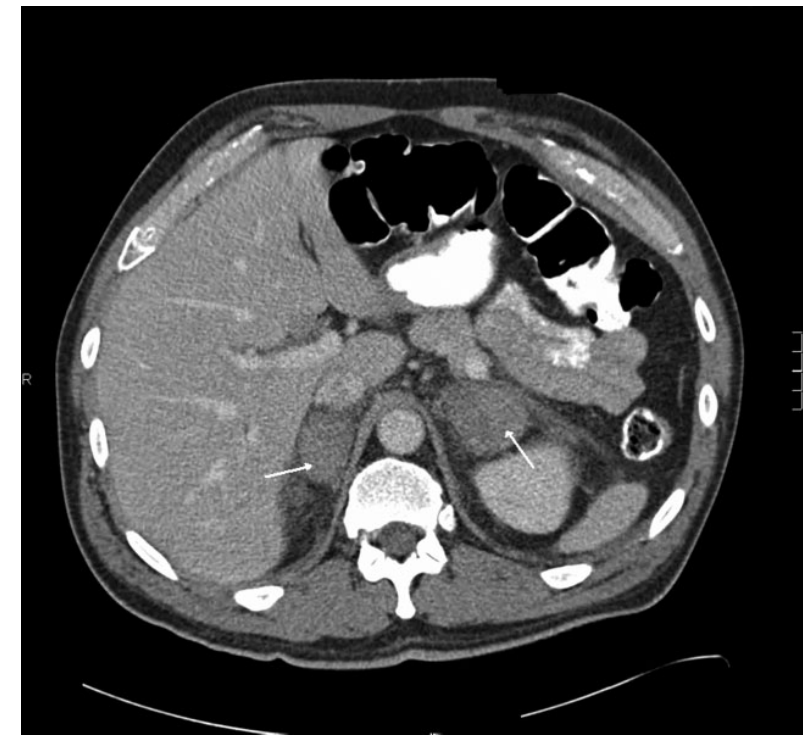

FIGURE 1. Bilateral adrenal hemorrhage on CT. Abbreviation: CT, computed tomography.

adrenal glands suggestive of bilateral acute adrenal hemorrhage. The enoxaparin was discontinued and empiric steroid replacement therapy was begun. A random cortisol level was normal but a cosyntropin stimulation test showed an absolute increase in cortisol level of only $0.8 \mu \mathrm{g} / \mathrm{dL}$ at both 30 and 60 minutes after administration of $250 \mu \mathrm{g}$ of cosyntropin. An investigation was undertaken to determine if the patient had any prior risk factors for bleeding. There was no evidence of infection and a comprehensive evaluation for bleeding, and coagulation disorders was normal. The bilateral adrenal hemorrhage was attributed to the use of enoxaparin in the postoperative setting. Unfortunately, the patient subsequently developed a deep venous thrombosis in his lower extremity and an inferior vena cava (IVC) filter was placed before discharge. He was doing well 6 months later, and is still continued on glucocorticoid and mineralocorticoid replacement therapy and follows up with endocrinology as an outpatient. 


\section{Discussion}

Bilateral adrenal hemorrhage is usually associated with massive sepsis from Gram-negative organisms such as Neisseria meningitides, Pseudomonas aeroginosa, Escherichia coli, and Bacteroides fragilis. Rupert Waterhouse, in 1911, was the first person to describe a patient with severe meningococcal sepsis resulting in acute adrenal hemorrhage and collapse. This was also later described independently by Carl Friderichsen in 1918, and is now referred to as the Waterhouse-Friderichsen syndrome. Other causes include antiphospholipid antibody syndrome, heparin-associated thrombocytopenia (HIT), and severe physical stress. Bilateral adrenal hemorrhage can also spontaneously occur in the postoperative period, especially after cardiothoracic or orthopedic surgery. This phenomenon may be related to the frequent use of prophylactic anticoagulants after these types of procedures.

The first case report of bilateral adrenal hemorrhage secondary to use of anticoagulants was described in 1947, and the first case report of successful resuscitation after corticosteroid administration in a patient with bilateral adrenal hemorrhage secondary to anticoagulant use was described by Thorn in $1956 .^{1}$ A review of the literature demonstrates multiple case reports of adrenal hemorrhage reported in the postoperative period, particularly after joint arthroplasty, and especially after knee replacement surgeries. Most of the recent cases have been associated with use of prophylactic low-dose heparin or low-molecular-weight heparin at the time of adrenal hemorrhage. In a study of 157 case reports of individuals with bilateral hemorrhage (including 22 autopsies), 48 cases were associated with administration of anticoagulants, although the dose and effect were not specified. ${ }^{2}$ Amador et al. ${ }^{1}$ showed that out of 4325 autopsies performed from 1949 to 1962 in their institution, 30 cases were found of bilateral hemorrhage, of which 10 were receiving heparin at presumably prophylactic doses; 5 of these patients were also receiving dicumarol.

Mayo Clinic investigators performed a retrospective review of all cases of adrenal hemorrhage over a period of 25 years at their hospital, and found 141 cases of adrenal hemorrhage, of which 78 were bilateral and 63 were unilateral, $^{3}$ and in 67 patients the condition was diagnosed at autopsy. In this study 14 patients had adrenal hemorrhage in the postoperative period in the absence of lupus anticoagulant or HIT; there was no specific mention in this study of the use of postoperative anticoagulants. Finally, a multicenter case control study was undertaken by Kovacs et al. ${ }^{4}$ to assess putative risk factors for development of bilateral massive adrenal hemorrhage. In the multivariate analysis, thrombocytopenia, exposure to heparin, and sepsis were found to be strongly associated with risk of hemorrhage. Of 23 patients with bilateral, massive adrenal hemorrhage, 16 had been exposed to heparin, and at least 6 were on exclusively subcutaneous heparin. The authors concluded that heparin exposure was a much bigger risk factor than other coagulopathies, and those exposed to heparin of any route or type for 4 to 6 days and those exposed for more than 6 days were about 17 and 34 times, respectively, more likely to develop bilateral hemorrhage than those who had less than 4 days or no exposure.

The clinical presentation of adrenal insufficiency due to bilateral adrenal hemorrhage is often nonspecific. Symptoms may include abdominal pain, back pain, fever, nausea, vomiting, weakness, obtundation, confusion, and hypotension-all of which are also common postoperative symptoms and can be missed or ignored. ${ }^{5}$ Rao et al. ${ }^{6}$ profiled the clinical presentation of 64 cases of bilateral hemorrhage and found the following: abdominal, flank, back, or chest pain (86\%); anorexia, nausea, or vomiting (47\%); psychiatric symptoms (42\%); fever (66\%); hypotension recognized before shock episode (19\%); and abdominal rigidity or rebound (22\%). Adrenal insufficiency becomes clinically evident once $90 \%$ of the gland is destroyed. About $50 \%$ of patients do not manifest typical laboratory abnormalities, so a high degree of suspicion is necessary to diagnose the condition. $^{3}$ Also, the laboratory diagnosis of adrenal insufficiency using random cortisol levels is unreliable, as reference ranges in patients experiencing stress (as in the postoperative period) have not been well studied or established. In patients with bilateral hemorrhage postoperatively on prophylactic anticoagulants, the coagulation profile is usually within normal limits and there is typically no evidence of spontaneous bleeding elsewhere. In later stages, the typical laboratory findings of abnormal adrenal function such as hypokalemia, hyponatremia, declining cortisol levels, and an inappropriate response to adrenocorticotropic hormone stimulation test may be seen. A significant drop in hemoglobin secondary to hemorrhage may also be encountered in some patients secondary to the bleed.

CT is the most reliable and extensively used imaging modality for making the diagnosis, although magnetic resonance imaging (MRI) or ultrasound may also be utilized. Early in the course of adrenal hemorrhage, CT findings may be negative, and repeated imaging is appropriate when clinical suspicion is high. The presence of bilateral adrenal enlargement with increased signal attenuation suggests bilateral adrenal hemorrhage. MRI can both characterize adrenal hematomas, and estimate their age.,

Postoperative adrenal hemorrhage and insufficiency is easily treatable and has excellent outcomes; survivors will need lifelong corticosteroid replacement (and usually mineralocorticoid replacement as well). In the Mayo Clinic study, survival was $100 \%$ with treatment vs. $17 \%$ without treatment. In comparison, sepsis-induced or stress-induced adrenal insufficiency has poor outcomes despite adequate treatment (9\% survival with treatment vs. $6 \%$ survival without treatment). ${ }^{3}$ Death can occur within hours to days of symptoms if untreated. Treatment includes timely initiation of adrenal hormone replacement and reversal of coagulopathies.

Postoperative venous thromboembolism (VTE) prophylaxis with anticoagulants is the appropriate care in many cases, but, along with the postoperative state itself, also appears to be a risk factor for this unusual condition. 
Postoperative bilateral adrenal hemorrhage is rare and potentially fatal. Early identification and prompt initiation of steroid replacement therapy and reversal of coagulopathies can prove to be lifesaving. Making this diagnosis can be very challenging, as the clinical presentation and laboratory findings of adrenal hemorrhage are vague and nonspecific and mimic many nonlife threatening post-operative complications. Radiological diagnosis by CT may initially be normal and thus further confound the diagnosis. Hence, providers should remain vigilant for associated complications even with low-dose prophylactic heparin or low-molecular-weight heparin in postoperative patients, and prompt, presumptive treatment with corticosteroids should be started while awaiting confirmation by imaging and laboratory testing.

\section{Address for correspondence and reprint requests:}

Anitha Rajamanickam, MD, 9500 Euclid Avenue, S70/Department of Internal Medicine, Cleveland, OH 44195; Telephone: 216-4458383; Fax: 216-444-8530; E-mail: rajamaa@ccf.org Received 27
December 2008; revision received 27 December 2008; accepted 7 January 2009.

\section{References}

1. Amador E. Adrenal hemorrhage during anticoagulant therapy. A clinical and pathological study of ten cases. Ann Intern Med. 1965;63(4):559-571.

2. Xarli VP, Steele AA, Davis PJ, Buescher ES, Rios CN, Garcia-Bunuel R. Adrenal hemorrhage in the adult. Medicine. 1978;57(3):211-221.

3. Vella A, Nippoldt TB, Morris JC 3rd. Adrenal hemorrhage: a 25-year experience at the Mayo Clinic. Mayo Clin Proc. 2001;76(2):161-168.

4. Kovacs KA, Lam YM, Pater JL. Bilateral massive adrenal hemorrhage. Assessment of putative risk factors by the case-control method. Medicine. 2001;80(1):45-53.

5. Rao RH. Bilateral massive adrenal hemorrhage. Med Clin North Am. 1995; 79(1):107-129

6. Rao RH, Vagnucci AH, Amico JA. Bilateral massive adrenal hemorrhage: early recognition and treatment. Ann Intern Med. 1989;110(3):227-235.

7. Kawashima A, Sandler CM, Ernst RD, et al. Imaging of nontraumatic hemorrhage of the adrenal gland. Radiographics. 1999;19(4):949-963.

8. Hoeffel C, Legmann P, Luton JP, Chapuis Y, Fayet-Bonnin P. Spontaneous unilateral adrenal hemorrhage: computerized tomography and magnetic resonance imaging findings in 8 cases. J Urol. 1995;154(5):1647-1651. 